\author{
Lorenzo Preda \\ Elvio De Fiori \\ Cristiano Rampinelli \\ Mohssen Ansarin \\ Giuseppe Petralia \\ Fausto Maffini \\ Daniela Alterio \\ Luke Bonello \\ Fausto Chiesa \\ Massimo Bellomi
}

\section{US-guided transcutaneous tru-cut biopsy of laryngo-hypopharyngeal lesions}

Received: 21 July 2009

Revised: 15 October 2009

Accepted: 29 October 2009

Published online: 17 December 2009

(C) European Society of Radiology 2009
L. Preda $(\bowtie) \cdot$ E. De Fiori .

C. Rampinelli · G. Petralia .

L. Bonello $\cdot$ M. Bellomi

Department of Radiology,

European Institute of Oncology,

Via Ripamonti 435,

20141 Milan, Italy

e-mail: lorenzo.preda@ieo.it

Tel.: +39-2-57489041

Fax: +39-2-57489040

M. Ansarin · F. Chiesa

Department of Head and Neck Surgery,

European Institute of Oncology,

Via Ripamonti 435,

Milan, Italy

F. Maffini

Patology Division,

Laboratory of Anatomy,

European Institute of Oncology,

Via Ripamonti 435,

Milan, Italy

\section{Alterio}

Radiotherapy Division,

European Institute of Oncology,

Via Ripamonti 435,

Milan, Italy

M. Bellomi

School of Medicine,

University of Milan,

Milan, Italy

\begin{abstract}
Objective: To evaluate the feasibility and performance of ultrasound-guided transcutaneous tru-cut biopsy (USGTCB) in selected patients (with stenosis of airways or difficult intubation or contraindication to general anaesthesia) with untreated or previously treated suspicious laryngo-hypopharyngeal masses. Methods: Biopsies were performed with a free-hand technique by a single radiologist. Thirty-six USGTCBs were scheduled in 34 patients (24 males, 10 females; age range $47-$ 95 years). Two USGTCBs were not performed, as lesions were not detectable: therefore, 16 USGTCBs were performed for an untreated mass suspicious for malignancy and 18 were performed for a mass suspicious
\end{abstract}

for recurrence after radiotherapy alone, or associated with endoscopic laser surgery or chemotherapy. Results: USGTCB diagnosed 25 squamous cell carcinomas $(73.5 \%)$ and nine benign lesions $(26.5 \%)$; no false positives and two false negatives were reported, both in patients previously treated with radiotherapy. The sensitivity, specificity, positive and negative predictive value of the technique was $92.5 \%, 100 \%, 100 \%$ and $77.7 \%$ respectively, with no major complications. Conclusion: Although biopsy under microlaryngoscopy remains the "gold-standard" technique, USGTCB is feasible, carries the advantages of avoiding general anaesthesia, is suitable for outpatients and is cost-effective. If applied to selected patients, it could be considered for the histological diagnosis of both primary and recurrent laryngo-hypopharyngeal masses.

Keywords Tru-cut biopsy · Laryngeal cancer - Ultrasound . Diagnosis · Histology

\section{Introduction}

Trans-cutaneous fine-needle aspiration cytology (FNAC) and tru-cut biopsy, performed under ultrasound (US) or computed tomography (CT) guidance, have an important role in the diagnostic work-up of the head and neck masses
[1-4]. However, the laryngeal space may not be easily accessible with a transcutaneous approach and biopsies of the masses in this district are usually performed by microlaryngoscopy, in order to obtain material for histological diagnosis and to assess the feasibility of conservative surgery. There are, however, some technical limitations to 
this approach: the intubation could be difficult in patients with large masses obstructing the upper airways and the biopsy procedure is often complicated by breathing difficulties due to haemorrhage or oedema. For such reasons, a protective tracheotomy may be required.

Trans-oral laryngeal biopsy under local anaesthesia is an alternative approach when a patient has a contraindication to the general anaesthesia; however, it may be difficult in patients with large masses obstructing the airways and it may be burdened with specimen inadequacy problems when performed for a suspected recurrence after radiotherapy (RT) or chemo-radiotherapy [5-7].

A pilot study from Ansarin et al. [5] showed that ultrasound-guided transcutaneuos tru-cut biopsy (USGTCB) is a feasible technique for the diagnosis of bulky laryngohypopharyngeal masses in patients who have a contraindication to general anaesthesia (e.g. patients at high risk for serious co-morbidity or multiple concomitant malignancies in the oro-pharyngeal area) and in patients who would not be candidates for conservative surgery if the mass was malignant, overcoming the disadvantages of FNAC. In fact, the cytological sample is unable to differentiate in situ from invasive carcinoma and is often inadequate or falsely negative in patients already treated with RT or chemoradiotherapy $[8,9]$.

The purpose of this study was to evaluate the feasibility of USGTCB in patients with untreated or previously treated suspicious laryngo-hypopharyngeal masses and to evaluate its performance for obtaining a histological diagnosis.

\section{Materials and methods}

\section{Patients}

This study was approved by our institutional ethics committee and a written informed consent was obtained from all patients prior to starting the study.

Between August 2004 and February 2008, patients with untreated or previously treated suspicious laryngo-hypopharyngeal masses were prospectively enrolled in the study if the following criteria were met: (1) stress dyspnoea due to a stenosis of airways, which would have required a protective tracheotomy for a microlaryngoscopy under general anaesthesia; (2) signs indicating difficult intubation $[10,11]$ or (3) relative contraindication to general anaesthesia; (4) CT or magnetic resonance imaging (MRI) of the head and neck performed within 1 month before the procedure.

Patients without a history of malignancy were included if they had a mass in the larynx or hypopharynx (only piriform sinus subsite) suspicious of malignancy that was staged as T3 or $\mathrm{T} 4$ at clinical and/or cross-sectional imaging by $\mathrm{CT}$ or MRI, or if they had bulky T2 lesions of the supraglottic larynx. Patients with a history of tumors in the larynx or hypopharynx, treated with RT, chemotherapy and/or surgery were included if they had a laryngo-hypopharyngeal mass suspicious of recurrence at clinical examination and/or CT or MRI follow-up.

Patients excluded were those with massively calcified thyroid cartilage, those with cT1 disease, those with superficial glottic cT2 lesions not detectable by ultrasound and those with an abnormal coagulation profile including prothrombin activity (PA), partial thromboplastin time (PTT) and international normalized ratio (INR), with normal values being respectively $70-120 \%, 17-26 \mathrm{~s}$, and $0.8-1.2$.

\section{Technique}

All biopsies were performed with the free-hand technique by a single radiologist (blinded for review) with 10 years experience in free-hand US-guided biopsies.

The first step of the USGTCB was the evaluation of the CT or MR images, to localize the mass candidate to the procedure and to have a panoramic assessment of the anatomic structures adjacent to the mass, in order to identify a potential path for the needle.

The second step was accurate patient positioning: all patients laid in the supine position on a bed reclined at $35^{\circ}$ and with the head (hyper)extended. The skin was disinfected with antiseptic and covered with a sterile drape.

The third step was the assessment by US of the mass in the larynx or hypopharynx, in order to evaluate: first, if the mass was visible with US and, second, if it was possible to identify a safe path for the needle. The US examination also included an assessment with the colour-Doppler technique, in order to identify vessels adjacent to the mass or eventually crossing the hypothesized path of the needle.

All the US exams, including colour-Doppler study, were performed on a Sequoia 512 USG machine (Siemens, Erlangen, Germany), using a multifrequency linear $15 \mathrm{~L} 9 \mathrm{~W}$ probe (Siemens, Erlangen, Germany).

If the mass was visible at US and judged as a candidate for the USGTCB, an 18-gauge cannula was placed in the antecubital vein and local anaesthesia $(10 \mathrm{ml}$ of $2 \%$ carbocaine) was performed in the tissues along the hypothesized path of the needle as well as in the subcutaneous tissues, using a 22-gauge spinal needle (Terumo Medical Corporation, Tokyo, Japan). Intravenous sedation or analgesia was never performed.

For the lesions at the supraglottic level, a path was selected for the needle through the thyrohyoid membrane; for the glottic and subglottic lesions a path through the cricothyroid ligament; for lesions of the piriform sinus a path lateral to the free edge of the thyroid cartilage. If the full thickness of the cartilage was infiltrated, the needle was introduced directly through it.

Once the path for the needle was selected, a 16- or 18-gauge spring-loaded biopsy needle (Biopsy-bell, Mirandola, Italy) was used for the biopsy under US guidance. 
At the end of the procedure the patients were kept at rest in the recovery room for $30 \mathrm{~min}$ and then discharged if no complications arose.

Tissue cores were placed in $10 \%$ phosphate-buffered formalin for $12 \mathrm{~h}$, paraffin embedded and sectioned at 4 $\mu \mathrm{m}$, followed by standard stain with hematoxylin and eosin ( $\mathrm{H} \& \mathrm{E})$; when required, a immunohistochemical stain was also performed, for a better evaluation of the neoplasm and comprehension of histological type.

\section{Results}

From the 40 patients who were canditates for the USGTCB, six $(15 \%)$ were excluded for the following exclusion criteria: two for massively calcified thyroid cartilage, four for the presence of a glottic cT2 disease.

Therefore, 36 USGTCBs were scheduled in 34 patients (24 males, 10 females; mean age 65 years, range 47-95 years); in two patients who met the inclusion criteria, the procedure was scheduled both for the first diagnosis and for a suspicious recurrence of squamous cell carcinoma (SCC), after RT in one case and after chemo-radiotherapy in the other.

Two USGTCBs were not performed in two patients (one with cT2 untreated supraglottic laryngeal mass and one with suspected T3 laryngeal recurrence), as lesions were not detectable by US. Therefore, a total of 34 USGTCBs were performed in 32 patients: 16 USGTCBs were performed for an untreated mass suspicious of malignancy, including 12 masses in the larynx (two cT2, seven cT3 and three cT4) and four masses in the piriform sinus (three cT3 and one cT4), and 18 USGTCBs were performed for a mass suspicious of recurrence (two cT2, eight cT3, eight cT4) after endoscopic laser surgery and RT in 13 cases, RT alone in three cases and chemo-radiotherapy in two cases. A 16-gauge core biopsy needle was used in 14 procedures and an 18-gauge core biopsy needle in 20 procedures. None of the enrolled patients developed cancer cell dissemination due to USGTCB. All the 34 specimens obtained with the USGTCB were sufficient for diagnosis. At pathology, lesions were diagnosed as invasive SCC $(73.5 \%)$ in 25 cases (true positives) (Fig. 1) and as benign in nine cases $(26.5 \%)$, including postradiotherapy fibrosis, in all cases, associated with chondronecrosis in two patients. Fifteen out of the 25 SCCs underwent subsequent surgery, which confirmed the diagnosis of malignancy; the remaining ten SCCs were treated with chemoradiotherapy and followed-up with clinical examination and $\mathrm{CT}$ or MRI.

The nine benign lesions were all diagnosed in patients previously treated with RT alone or associated with endoscopic laser surgery or chemotherapy; in seven of them (true negatives) (Fig. 2), the diagnosis was confirmed by surgery in two cases and by follow-up (mean follow-up 22 months, range 13-28 months) in five cases, whereas in the remaining two cases (false negatives) (Fig. 3) a subsequent surgical biopsy did not confirm the diagnosis performed with the tissue cores from the USGTCB, showing the presence of SCC. No false positive result was reported.

Overall, the sensitivity of the technique was $92.5 \%$; the specificity was $100 \%$; positive and negative predictive values were $100 \%$ and $77.7 \%$, respectively.

Sensitivity, specificity, positive and negative predictive value for the 18 procedures with the suspicion of recurrence were $81.8 \%, 100 \%, 100 \%$ and $77.7 \%$, respectively.

The time required for the USGTCB ranged from 15 to 24 min (mean time $18 \mathrm{~min}$ ). We did not observe major complications, according to the categorization of the Society of Interventional Radiology [12]. One patient, who underwent USGTCB for the suspicion of laryngeal recurrence 2 years after RT, developed a near-syncope, which resolved spontaneously.
Fig. 1 A patient with suspected recurrence of SCC of the larynx after RT. a CT shows an enhancing lesion of the right vocal cord causing wide erosion of the thyroid cartilage and involvement of the adjacent soft tissue. b USGTCB obtained using a semiautomated 18-gauge biopsy needle (white arrow), which has been introduced into the lesion through the disrupted thyroid cartilage
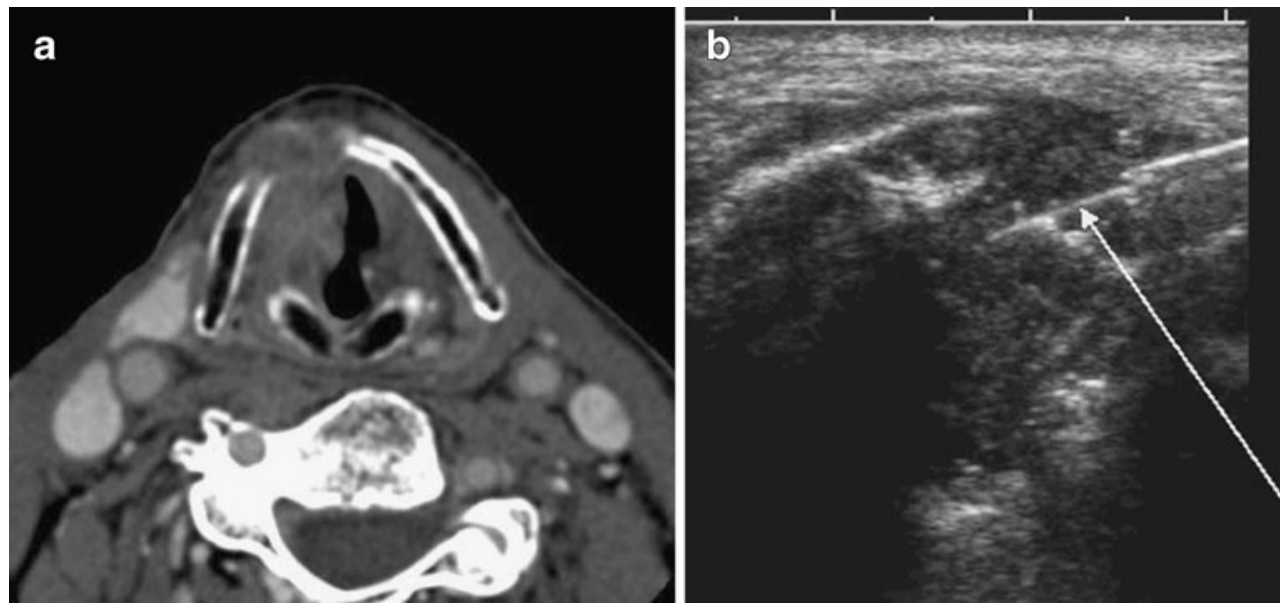
Fig. 2 A patient with clinical suspicion of recurrence in the glottic-subglottic region after endoscopic laser surgery and RT. a CT at the level of the subglottis, showing enhancing soft tissue anteriorly causing airway stenosis. b US image obtained in the same plane, showing the needle tip within the lesion (markers 1 and 2 indicating calipers used to measure the lesion). Histopathology was compatible with fibrotic tissue associated with inflammatory changes. The diagnosis was then confirmed by endoscopic surgery
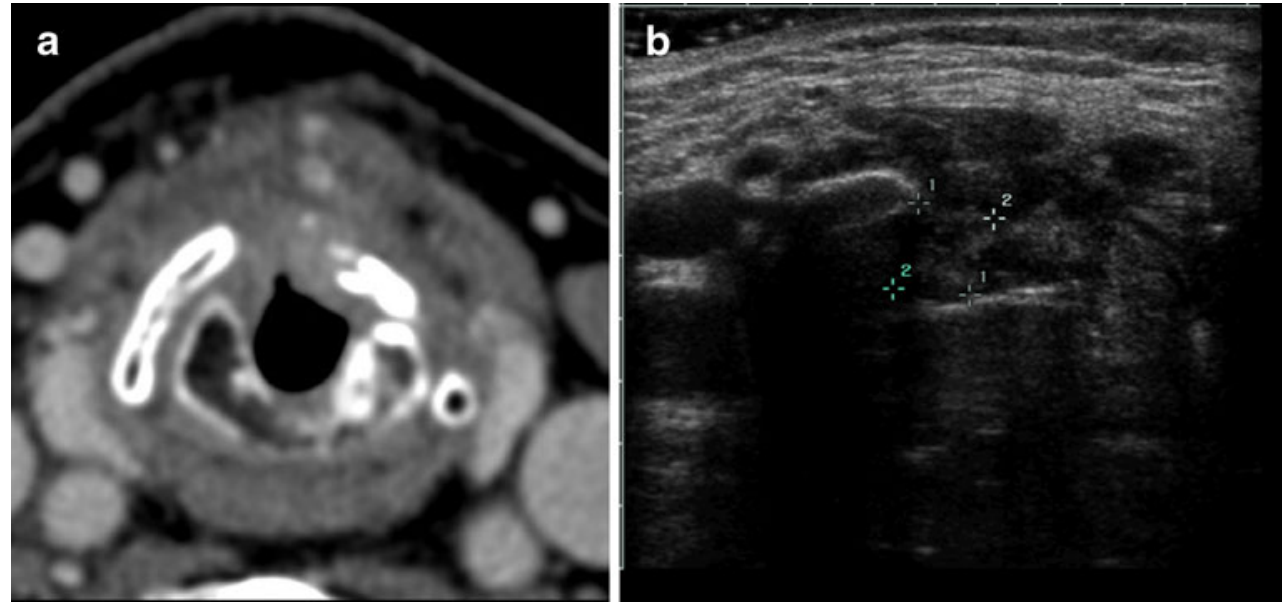

\section{Discussion}

The biopsy obtained by microlaryngoscopy under general anaesthesia is the diagnostic approach of choice in patients with a suspected laryngo-hypopharyngeal mass.

In clinical practice there are categories of patients to whom the use of such a diagnostic procedure is risky or charged by an elevated percentage of false negatives $[13,14]$.

On the basis of the pilot study by Ansarin et al. [5], we have prospectively enrolled for USGTCB selected patients with laryngo-hypopharyngeal masses newly diagnosed or suspicious of recurrence after RT alone or associated with surgery or chemotherapy. USGTCB was feasible in 32 out of the 34 patients who met the inclusion criteria and were scheduled for the procedure. The rigid selection of the patients candidate to the USGTCB and the accurate review of the previous radiological examinations (CT or MRI) prior to the procedure may account for the feasibility of the procedure observed in our series. In the two patients who met the inclusion criteria (one with untreated cT2 lesion and the other with suspected T3 glottic recurrence after RT) but did not undergo USGTCB, the procedure was not feasible, as in both cases the mass was not detectable due to a limited acoustic window, caused by a calcified thyroid cartilage. The thyroid cartilage is the most important anatomical obstacle in patients referred for USGTCB, especially in adults, in whom it is largely calcified and could not easily be crossed by the needle. In those cases, in order to reach the lesions it is necessary that the area of

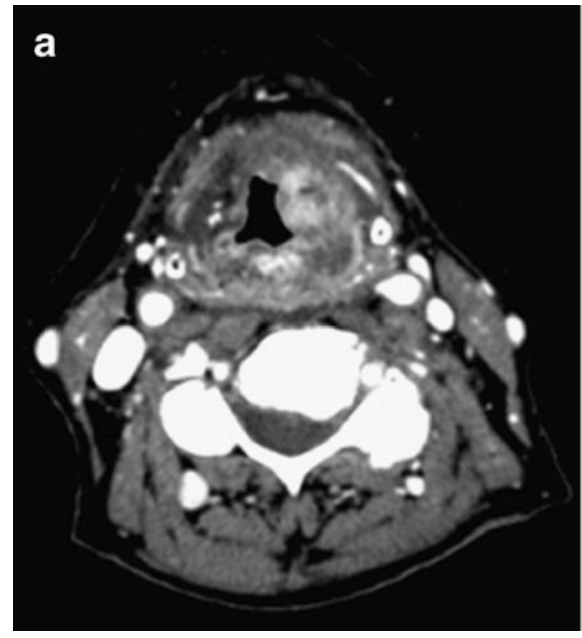

Fig. 3 A patient who was treated 1 year before with chemoradiotherapy for laryngeal SCC, presenting with swelling of the left false vocal cord region. a CT at the supraglottic level showing a submucosal soft tissue mass with inhomogeneous enhancement on the left side suspicious for tumour recurrence. b US image showing

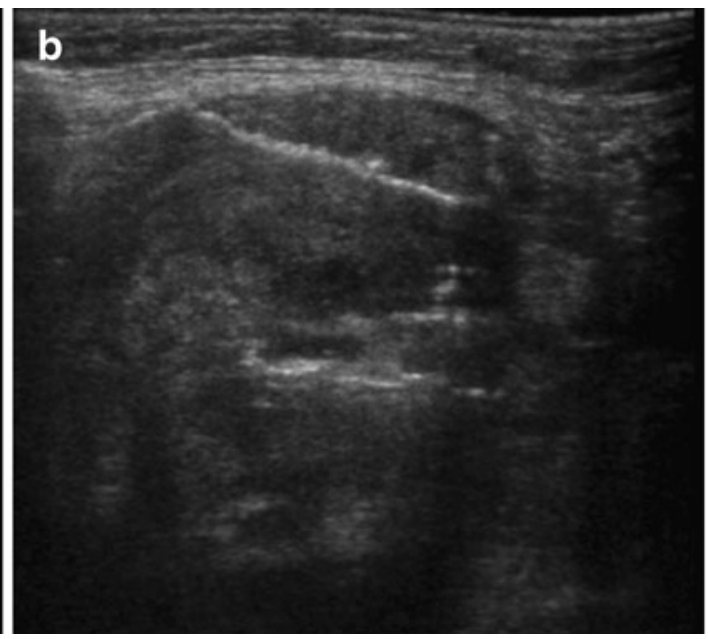

the tip of the tru-cut needle on the left side of the supraglottis. Histopathology was inconclusive for tumour recurrence. The final diagnosis of recurrence was obtained by multiple biopsies under microlaryngoscopy during general anaesthesia and the patient then underwent total laryngectomy 
erosion due to tumour spread is sufficiently wide to obtain a proper sample.

The long experience of the radiologist that performed all the USGTCBs in this study may have contributed to the feasibility of the procedure. We believe that an extensive knowledge of the regional anatomy and a suitable experience in the execution of free-hand biopsy procedures under US guidance are mandatory to safely perform USGTCB. When the procedure was feasible amongst the eligible patients with an untreated laryngeal-hypopharyngeal mass (in 16 patients), the samples were diagnostic in all cases.

The choice of the needle size, 16- or 18-gauge, was based upon the dimension of the lesion and the size of the acoustic window.

When the USGTCB was performed on masses suspicious for recurrence, two false-negative results out of 18 procedures were observed, with a sensitivity of $81.8 \%$. Both false-negative patients, however, had a previous history of radiotherapy treatment and it is known that previous (chemo)radiotherapy may impair the diagnosis of recurrence and that the most reliable technique to obtain a histological diagnosis would be via microlaryngoscopy under general anaesthesia [15]. The assessment of recurrent laryngeal carcinoma after (chemo)radiotherapy is much more difficult than the assessment of "de novo" carcinoma either by endoscopy or by imaging studies for various reasons: tumour may recur after radiation therapy in multicentric foci, undetectable by imaging studies, residual inflammation changes may be associated with radiotherapy, tumour recurrence may be localised submucosally and invisible during endoscopy [16]. Often, symptoms and signs for tumour recurrence and radionecrosis are identical and patients undergo numerous inconclusive endoscopy procedures [17]. In many of these cases the radiological findings typical for chondro-radionecrosis, such as progressive crico-arytenoid sclerosis, presence of gas bubbles as well as fragmentation and collapse of cartilages, are not present [18]. In contrast, the appearance of areas of focal contrast enhancement not present at prior imaging exams may be suspicious but not specific for tumour recurrence $[17$, 19]. Although some authors recommend ${ }^{18} \mathrm{~F}$-fluorodeoxyglucose positron emission tomography $\left({ }^{18}\right.$ F-FDG-PET) for post-treatment imaging [20], its value is currently not firmly established [21], as possible false-positive results may occur because of therapy-induced inflammatory changes and false-negative results may occur due to its low spatial resolution [22]. Diffusion-weighted MRI (DW-MRI) has shown in initial experiences a high accuracy for tissue differentiation in the post-RT phase independently from inflammatory or necrosis-induced tissue distortions [21]. Since both modalities are able to diagnose a recurrence at an early stage, they can potentially avoid delay of treatment, reducing the risk of disease progression and, therefore, improving patient management. However further studies, ideally with a larger patient population are needed.

In our two false-negative cases, CT was able to show focal areas of contrast enhancement greater than $1 \mathrm{~cm}$ within the original tumour bed, involving the glottic region; the diagnosis of recurrence was obtained in both cases with multiple biopsies by microlaryngoscopy under general anaesthesia. The two patients then underwent total laryngectomy, with the post-operative diagnosis of pT2 and pT3 SCC.

In conclusion, in our study USGTCB was a feasible technique, which has the advantages of avoiding general anaesthesia, being suitable for outpatients and being costeffective when compared with the alternative techniques. In our study, USGTCB provided adequate specimens for histological analysis, including immunohistochemical methods, thus showing good performance for obtaining a histological diagnosis of untreated or previously treated laryngo-hypopharyngeal masses suspicious of malignancy. If it were applied to selected patients, USGTCB could be considered for the histological diagnosis of both primary and recurrent hypopharyngeal and laryngeal masses before performing the biopsy under general anaesthesia using microlaryngoscopy.

\section{References}

1. Fulciniti F, Califano L, Zupi A, Vetrani A (1997) Accuracy of fine needle aspiration biopsy in head and neck tumors. J Oral Maxillofac Surg 55:1094-1097

2. Jandu M, Webster K (1999) The role of operator experience in fine needle aspiration cytology of head and neck masses. Int J Oral Maxillofac Surg 28:441-444

3. Dedivitis RA, De Carvalho MB, Rapoport A (2000) Transcutaneous fine needle aspiration biopsy of the preepiglottic space. Acta Cytol 44:158-162
4. Amedee RG, Dhurandhar NR (2001) Fine-needle aspiration biopsy. Laryngoscope 111:1551-1557

5. Ansarin M, De Fiori E, Preda L, Maffini F, Bruschini R, Calabrese L, Jereczek-Fossa BA, Chiesa F, Bellomi M (2007) Ultrasound-guided transcutaneous tru-cut biopsies to diagnose laryngopharyngeal masses. A pilot study. Cancer 109:2268-2272

6. Bahadur S, Amatya RC, Kacker SK (1985) The enigma of postradiation oedema and residual or recurrent carcinoma of the larynx and pyriform fossa. J Laryngol Otol 99:763-765
7. O’Brien PC (1996) Tumour recurrence or treatment sequelae following radiotherapy for larynx cancer. J Surg Oncol 63:130-135

8. Seetharam SS, Ramachandran CR (1998) Fine needle aspiration cytology as a diagnostic test for oral squamous cell carcinoma. Oral Dis 4:180-186

9. Pisharodi LR (1997) False-negative diagnosis in fine-needle aspirations of squamous-cell carcinoma of head and neck. Diagn Cytopathol 17:70-73 
10. Mallampati SR, Gatt SP, Gugino LD, Desai SP, Waraksa B, Freiberger D, Liu PL (1985) A clinical sign to predict difficult tracheal intubation: a prospective study. Can Anaesth Soc J 32:429434

11. Langeron O, Amour J, Vivien B, Aubrun F (2006) Clinical review: management of difficult airways. Clin Care 10:243-247

12. Sacks D, McClenny TE, Cardella JF, Lewis CA (2003) Society of Interventional Radiology clinical practice guidelines. J Vasc Interv Radiol 14: S199-S202

13. Citardi MJ, Abrahams JJ, Flynn S, Sasaki CT (1996) Efficacy of transcutaneous computed tomography-guided fine needle aspiration biopsy in patients with laryngeal squamous cell carcinoma, probable failed radiation therapy, and negative transmucosal biopsies. Laryngoscope 106:1244-1247
14. Brouwer J, Bodar EJ, De Bree R et al (2004) Detecting recurrent laryngeal carcinoma after radiotherapy: room for improvement. Eur Arch Otorhinolaryngol 261:417-422

15. Brouwer J, Hooft L, Hoekstra OS, Riphagen II, Castelijns JA, de Bree R, Leemans CR (2008) Systematic review: accuracy of imaging tests in the diagnosis of recurrent laryngeal carcinoma after radiotherapy. Head Neck 30:889-897

16. Zbären P, Nuyens M, Curschmann J, Stauffer E (2007) Histologic characteristics and tumor spread of recurrent glottic carcinoma: analysis on whole-organ sections and comparison with tumor spread of primary glottic carcinomas. Head Neck 29:26-32

17. Zbären P, Caversaccio M et al (2006) Radionecrosis or tumor recurrence after radiation of laryngeal and hypopharyngeal carcinomas. Otolaryngol Head Neck Surg 135:838-843

18. Hermans R, Pameijer FA, Mancuso AA, Parsons JT, Mendenhall WM (1998) CT findings in chondroradionecrosis of the larynx. AJNR Am J Neuroradiol 19:711-718
19. Hermans R, Pameijer FA, Mancuso AA, Parsons JT, Mendenhall WM (2000) Laryngeal or hypopharyngeal squamous cell carcinoma: can follow-up CT after definitive radiation therapy be used to detect local failure earlier than clinical examination alone? Radiology 214:683-687

20. Mukherji SK, Wolf GT (2003)

Evaluation of head and neck squamous cell carcinoma after treatment. AJNR Am J Neuroradiol 24:1743-1746

21. Vandecaveye V, De Keyzer F, Nuyts S, Deraedt K, Dirix P, Hamaekers P, Vander Poorten V, Delaere P, Hermans R (2007) Detection of head and neck squamous cell carcinoma with diffusion weighted MRI after (chemo)radiotherapy: correlation between radiologic and histopathologic findings. Int J Radiat Oncol Biol Phys 67:960-971

22. Hermans R (2008) Posttreatment imaging in head and neck cancer. Eur J Radiol 66:501-511 